\title{
Experimental techniques in hadron spectroscopy
}

\author{
P. Gianotti ${ }^{1, a}$ \\ ${ }^{1}$ INFN, Laboratori Nazionali di Frascati, via E. Fermi 40, 00044 Frascati, Italy
}

\begin{abstract}
Quantum Chromodynamics (QCD) is the theory of the strong interaction, but the properties of the hadrons cannot be directly calculated from the QCD Lagrangian and alternative approaches are then used. In order to test the different models, precise measurements of hadron properties are of extreme importance. This is the main motivation for the hadron spectroscopy experimental program carried out since many years with different probes and different detectors. A survey of some recent results in the field is here presented and commented, together with the opportunities offered by the forthcoming experimental programs.
\end{abstract}

\section{Introduction}

Quantum ChromoDynamics (QCD) is the theory of strong interaction that binds quarks and gluons to form hadrons. QCD is a non-linear theory that is not analytical. This implies that we are not able to solve the equations that derive from the QCD Lagrangian. At high energies, in order to describe strong interactions, perturbative approaches are successful, but in the low energy domain, to predict the properties of the hadrons, we have to use models.

Strong interaction is under study since long time, but recent findings of new and unexpected resonances show that the hadron spectrum is not yet completely understood. This is also underlined by the ongoing discussion on multi-quark states, and other exotic states with gluonic degrees of freedom.

Indipendentely from the theoretical framework used, it is undoubtful that not only mesons and baryons are possible. Other types of hadronic matter, multi-quark states or particles in which gluons contribute to the overall quantum numbers, should exist. Furthermore, all theoretical models rely on approximations and/or free parameters that must be constrained. This is why an intense activity in the hadron spectroscopy field is carried out since many years in different laboratories using different tools. The main goal is that to give an answer to the following questions:

- How we recognize non $q \bar{q}$ states?

- Can we observe experimentally gluonic degrees of freedom?

- How would these manifest themselves in terms of the excitation spectrum and also in the strong decays of hadrons?

A lot of information has been already collected and the complete puzzle is becoming more clear. Nevertheless, some unexpected and contradicting findings point out that we need to identify precisely all the physical states and their quantum numbers, and measure their masses and widths. We must

\footnotetext{
${ }^{\mathrm{a}}$ e-mail: paola.gianotti.lnf.infn.it
} 
determine the decay modes and branching ratios and study the underlying dynamics of production and decay. Only with such a complete set of experimental data, we can help guiding and constraining the theory. In the following sections I will briefly describe the analysis methods used and present an overview of some recent measurements performed in the hadron spectroscopy field.

\section{Partial Wave Analysis}

The most used tool to perform analyses of complex final states is Partial Wave Analysis (PWA). Within this framework a multi-particle phase space is expanded into a (truncated) series of angular momentum functions which describe the reaction matrix element $[2,3]$. The matrix element $M$ of some "decay operator" $U$ between an initial $i$ and a final $f$ state is written:

$$
M=<f|U| i>=<f\left|U_{A} U_{B} \ldots .\right| i>
$$

as the sum of all possible decays $(A, B$, etc...). The goal is to learn something about $U$ parameterizing $M$, and to determine the fitting parameters from the data. These parameters are the coefficients of "partial waves". To write down these waves, assumptions have to be made on how particles are grouped to construct the decay/reaction chain. The most used approach is the isobar model. It assumes that all sequences of decays appear to be two-body reactions. If we consider a particle 1 that has angular momentum quantum numbers $J$ and $M$ and that decays in two particles 2 and 3 having helicities $\lambda_{2}$ and $\lambda_{3}$ we can write:

$$
M=<f|U| i>=<2+3|U| 1>=<\theta, \phi, \lambda_{2}, \lambda_{3}|U| J M>
$$

having parameterized the interaction $U$ for the decay of a particle of spin $J$ into daughter particles with helicities $\lambda_{2}$ and $\lambda_{3} . \theta$ is the angle for the angular decay distribution and $\phi$ defines the phase convention. The best fit values of the free parameters are determined using an extended maximum likelihood technique or minimizing the $\chi^{2}$ of a set of histograms.

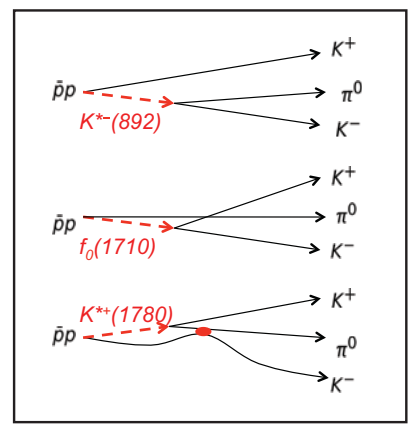

Figure 1: Example of the $K^{+} K^{-} \pi^{0}$ final state originated in the $\bar{p} p$ annihilation via different reactions.

As a practical example let us consider the $\bar{p} p$ annihilation reaction illustrated in fig. 1. The two charged kaons we see in the final state can be the decay products of an intermediate resonance (i.e. $f_{0}(1710)$ ), or we can have a $K^{+}$produced together with a $K^{*-}(892)$ that subsequently decay into $K^{-}+\pi^{0}$. The PWA technique helps to find out what happens in between the initial and the final state. 
ICNFP 2014

\begin{tabular}{ll}
\hline$f_{0}(980)$ & $4 q$ state, molecule \\
$f_{0}(1500)$ & $0^{++}$glueball \\
$f_{0}(1370)$ & $0^{++}$glueball \\
$f_{0}(1710)$ & $0^{++}$glueball \\
$\eta(1400) ; \eta(1460)$ & $0^{-+}$glueball \\
$f_{1}(1420)$ & $1^{++}$hybrid, $4 q$ state \\
$\pi_{1}(1400)$ & $1^{-+}$hybrid \\
$\pi_{1}(1600)$ & $1^{-+}$hybrid \\
$\pi(1800)$ & $0^{-+}$hybrid \\
$\pi_{2}(1900)$ & $2^{-+}$hybrid \\
$\pi_{1}(2000)$ & $1^{-+}$hybrid \\
$a_{2}^{\prime}(2100)$ & $1^{++}$hybrid \\
$\phi(2170)$ & $1^{--}$hybrid, $4 q$ state \\
\hline
\end{tabular}

Table 1: Main non- $q \bar{q}$ candidates in the light energy sector. In red states with exotic quantum numbers.

Nowadays, all the experiments studying hadron spectroscopy are using PWA techniques for the identification of the resonances and for the unambiguous determination of their properties: quantum numbers, pole positions, masses and widths. In the following sections I will show and comment some results of these activities.

\section{The low energy sector}

Thanks to the great variety of facilities available all over the world, in the last 30 years a wide harvest of data, in the mass range between 1 and $2 \mathrm{GeV} / c^{2}$, has been collected with the intention to search for exotic hadrons, i.e. hybrids, glueballs, multi-quark states predicted by the theory. Table 1 gives a list of states for which an exotic interpretation has been claimed.

Within exotics, there are states that are, in principle, easier to identify, since they exhibit non- $q \bar{q}$ quantum numbers. $\pi_{1}(1600)$, having quantum numbers $J^{P C}=1^{-+}$, is one of these, but its existence is strongly disputed within the community since the analyses done up to now are not conclusive and sometime they are not in agreement [4]. It has been identified for the first time in the $3 \pi$ final state from $\pi^{-} N$ interaction (BNL E852 and Protvino VES), and then confirmed in $\bar{p} p$ annihilation (CERN Crystal Barrel) [5-9]. The analyses performed by these collaborations were controversial since they were incomplete or relying on poor statistics. Recently, the COMPASS collaboration has published a new analysis of a sample of $420.000 \pi^{-} \pi^{-} \pi^{+}$events from the diffractive reaction

$$
\begin{array}{r}
\pi^{-}+P b \rightarrow X+(P b)_{\text {reco }} \\
\left\llcorner\pi^{-} \pi^{-} \pi^{+}\right.
\end{array}
$$

at $190 \mathrm{GeV} / c$ beam momentum[10] reporting a significant fraction $(1.7 \pm 0.2) \%$ of the $\pi_{1}(1600)$ exotic wave decaying to $\rho \pi$. This is coming out from an accurate PWA performed within the framework of the isobar model and including all known isovector and isoscalar $\pi \pi$ resonances in the fit. In total, 42 partial waves are included in the first step of the fit, while, in a second step, a $\chi^{2}$ fit of the spin-density matrix elements obtained for each mass bin in the first step is performed in the mass range from 0.8 

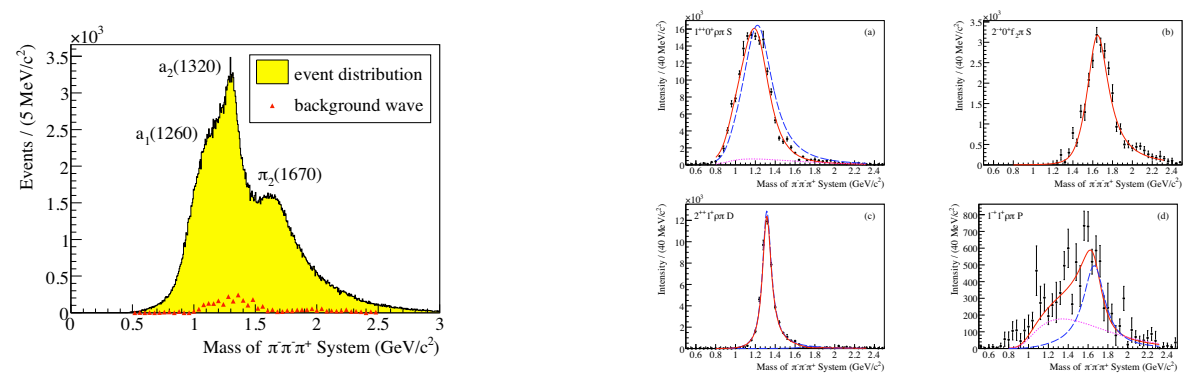

Figure 2: (left) Invariant mass of the $3 \pi$ system for $0.1 \mathrm{GeV}^{2} / c^{2}<\mathrm{t}^{\prime}<1.0 \mathrm{GeV}^{2} / \mathrm{c}^{2}$ (histogram), and intensity of the background wave with a flat distribution in 3-body phase space (triangles), obtained from a partial wave analysis in $40 \mathrm{MeV} / c^{2}$ bins of the $3 \pi$ mass and rescaled to the binning of the histogram (from [10]). (right) Intensities of major waves $1^{++} 0^{+} \rho \pi \mathrm{S}(\mathrm{a}), 2^{-+} 0^{+} \mathrm{f}_{2} \pi \mathrm{S}$ (b), and $2^{++} 1^{+} \rho \pi \mathrm{D}(\mathrm{c})$, as well as the intensity of the exotic wave $1^{-+} \rho \pi \mathrm{P}(\mathrm{d})$, as determined in the fit in mass bins (data points with error bars). The lines represent the result of the mass-dependent fit (from [10]).

to $2.32 \mathrm{GeV} / \mathrm{c}^{2}$. Figure 2(right) displays the intensities of the three more prominent waves resulting from the analysis, together with the exotic $1^{-+} 1^{+} \rho \pi \mathrm{P}$ wave.

Despite the COMPASS result, the hadron physics community is not yet convinced that such a state exists. This is due to the fact that similar searches led to different results. For instance, at the Jefferson Laboratory, the CLAS experiment has performed a PWA on a sample of 83.000 events of the reaction $\gamma p \rightarrow \pi^{+} \pi^{+} \pi^{-}(n)$ [11] with the intention to confirm the COMPASS result, but the outcome was eventually different. The analysis shows production of the $a_{2}(1320)$ and of the $\pi_{2}(1670)$ mesons, but no evidence for the $a_{1}(1260)$ and the $\pi_{1}(1600)$ exotic state has been found. I do not want to enter into the details of these analyses. I just want to stress their complexity that is paradigmatic to understand why the search for non- $q \bar{q}$ state is so hard. The signal of the exotic meson is not evident from the invariant mass plot. It comes out only after complicated and difficult to control analysis procedures, and this feeds skepticism and disputes. The production and decay amplitudes can be described with different spin formalisms; the complete input settings like the list of the hypotheses, the choice of the spin formalism and the description of the dynamics are subjective and there is no general consensus on which parameterization is best.

In the low energy domain, a new experiment that aims to map the complete spectrum of exotic hybrid mesons using photo-production is GlueX [12]. The GlueX detector will take data in a new experimental area (Hall D) built as part of the $12 \mathrm{GeV}$ upgrade project of the Jefferson Laboratory [13].

Few data on meson photo-production exist up to now, but recent theoretical calculations predict that hybrids would be produced in photo-production at roughly the same rate of ordinary mesons [14]. In addition, the availability at Jefferson Laboratory of linearly polarized photons will provide an extra tool to increase the sensitivity to resonance properties. For a given produced resonance, linear polarization will enable to distinguish the nature of the exchanged particles. This could be a powerful tool when performing PWA. Figure 3 shows the layout of the photon tagger and of the GlueX detector that is expected to start the data taking in 2015. This is a high acceptance, fixed target experiment consisting of a $2.2 \mathrm{~T}$ super-conducting solenoid, instrumented with different components, and of a 


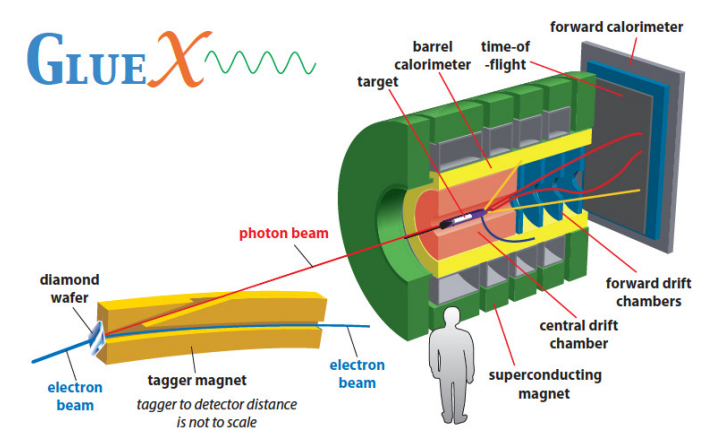

Figure 3: Layout of the GlueX experiment installed in Hall D of the Jefferson Laboratory.

set of detectors in the forward region. Charge particle tracking will be performed by means of a cylindrical drift chamber, made of straw tubes, and by four layers of planar chambers with cathode and strip readout, placed downstream. Neutral particles will be detected with a barrel calorimeter, made of lead and scintillating fibers, and with a forward module consisting of lead-glass blocks. A Time Of Flight system placed in the forward region, completes the setup.

\section{The charmonium energy sector}

Looking in the Particle Data Book [15], we see that in the mass region $1 \div 2 \mathrm{GeV} / \mathrm{c}^{2}$ there are hundreds of states. The average level spacing of mesons is $15 \mathrm{MeV} / \mathrm{c}^{2}$ while the average width is $150 \mathrm{MeV} / \mathrm{c}^{2}$. This implies superpositions and interferences that makes the experimental spectroscopy a very difficult job. This is another reason explaining why many states have been considered exotic candidates, but none of them is unambiguously assigned to the category.

In order to overcome these limitations, the spectroscopy activity moved to higher energies. Large data sets have been collected at $e^{+} e^{-}$machines by BaBar, Belle, CLEO, and BES collaborations. On the side of hadronic machines, the Tevatron experiments CDF and D0 have contributed significantly, and nowadays excellent data are coming form the LHC experiments.

In the sector of heavy quarks, the density of states is not so high and particle widths are narrower, then interference problems should be reduced. In particular, in the charmonium energy range only eight states are present below the $D \bar{D}$ threshold, and their width is in the $\mathrm{MeV} / \mathrm{c}^{2}$ range. Actually, the outcome of the spectroscopy studies performed in this energy range, has been extremely important. Thanks to this activity, it has been possible to prove the existence of exotic states. In fact, a series of new charged resonances has been identified whose quark composition cannot simply be $q \bar{q}$.

Nowadays, the set of new particles that are not fitting the conventional scheme, the so called " $X Y$ $Z$ states", is quite large. These mesons are a class of hadrons that are seen to decay to final states that contain a heavy quark and a heavy antiquark, but that cannot be assigned to any unseen $q \bar{q}$ level [16].

The first non- $q \bar{q}$ particle discovered in this energy range is the $X(3872)$ [17]. It has been identified in 2003 by the Belle collaboration in the reaction chain:

$$
\begin{aligned}
B^{+} \rightarrow & X(3872)+K^{+} \\
& \left\llcorner J / \psi+\pi^{+} \pi^{-}\right.
\end{aligned}
$$


and then intensively studied by several experiments. Since its discovery, it triggered many speculations about its nature since it could not be any of the expected unseen charmonium excited states. The proximity of its mass to the $D^{* 0} \bar{D}^{0}$ threshold has been a source of speculation on its nature. There are claims that this could be a $D^{* 0}-\bar{D}^{0}$ molecule, but to say more on this option, precise determinations of the particle characteristics are still needed. Furthermore, to establish whether $X(3872)$ is below or above $D^{* 0} \bar{D}^{0}$ threshold also a more accurate measurements of the $D^{0}$ mass is required.

Concerning the quantum numbers of $X(3872)$, the $\mathrm{LHCb}$ collaboration has recently established that the values of the total angular momentum, parity, and charge-conjugation eigenvalues are $1^{++}$ [18]. This has been achieved through a complete analysis of the full five-dimensional angular correlation between final state decay particles using a likelihood-ratio test.

About the mass, performing over-constrained fits, values below detector resolutions have been determined by many collaborations [19-22]. The mean value obtained combining all the available measurements for the $J / \psi \pi^{+} \pi^{-}$final state is $3871.68 \pm 0.17 \mathrm{MeV} / c^{2}$. This is slightly different from the value obtained studying $D^{* 0} \bar{D}^{0}$ final state $\left(3874.4 \pm 0.7 \mathrm{MeV} / c^{2}\right)$, and this has triggered speculations on the possibility that two states, very close in mass, could exit.

For the width, up to now, there is just an upper limit: $<1.2 \mathrm{MeV} / c^{2}$ [23], and it will be difficult for all present experiments to better determine it.

The $X(3872)$ precise width measurement will be one of the key features of the forthcoming PANDA experiment. $\bar{P}$ ANDA (antiProton ANnihilation at DArmstadt) [24] is a hadron spectroscopy experiment in preparation for the new Facility for Antiproton and Ion Research (FAIR) of Darmstadt [26]. $\overline{\mathrm{P}}$ ANDA will use a high intensity antiproton beam $\left(2 \times 10^{7} \bar{p} / \mathrm{s}\right)$ width high momentum resolution $\left(\delta p / p \simeq 2 \times 10^{-5}\right)$ to perform resonance scans. The technique consists in measuring the decay rate of a state, varying the energy of the beam in small steps [25]. With this method, the masses and the widths of all charmonium states could be measured with excellent accuracy, thanks to the precise knowledge of the initial $p \bar{p}$ state, and without any limitation due to the detector resolution. Furthermore, it would be also possible to evaluate the line shape of the resonances measuring several decay modes at the same time. For the $X(3872)$ by measuring $J / \psi \pi^{+} \pi^{-}$and $D^{* 0} \bar{D}^{0}$ final states, it would be possible to check whether there is one or two states close in mass and to determine their nature. Figure 4 shows the results of an energy scan simulation performed by the PANDA collaboration for the channel $X(3872) \rightarrow J / \psi \pi^{+} \pi^{-}$with subsequent $J / \psi \rightarrow e^{+} e^{-}$decay. Data points have been simulated varying the antiproton energy by $0.5 \mathrm{MeV}$ and assuming a peak cross section of $50 \mathrm{~ns}$ [27]. The input values for the particle mass and width are $3782 \mathrm{MeV} / \mathrm{c}^{2}$ and $100 \mathrm{keV} / c^{2}$, respectively. A four pion background has been also considered.

The $\bar{P}$ ANDA experiment will also have another big advantage. In $p \bar{p}$ annihilation it is possible to produce all final states that have $q \bar{q}$ quantum numbers, unlike $e^{+} e^{-}$interactions where only $1^{--}$final states can be directly accessed. Here, all other quantum numbers can be accessed only via secondary decays, and this limits the mass resolution achievable. Furthermore in $p \bar{p}$ annihilation, final states with "exotic" quantum numbers (e.g. non- $q \bar{q})$, can be directly formed if a recoil particle will be produced at the same time. As an example, PANDA will be able to study all the chargeed $Z$ states lying in the energy range from 2 to $5.5 \mathrm{GeV}$.

The first of such states has been found in the $\pi^{+} \psi^{\prime}$ invariant mass distribution by the Belle collaboration, studying $B \rightarrow K \pi^{+} \psi^{\prime}$ decay [28]. If this peak, called the $Z(4430)$, is interpreted as a meson, its minimum quark content should be $\mid c \bar{c} u \bar{d}>$. The LHCb collaboration, in a recent paper, has confirmed this discovery and has determined its $J^{P}$ values to be $1^{+}$[29].

By using alternatively the production reaction, $\bar{p} p \rightarrow Z^{ \pm} \pi^{\mp}$, or the formation one, $\bar{p} d \rightarrow$ $Z^{-} p_{\text {spectator }}$, a complete analysis of this new particle will be performed by the PANDA collaboration. 


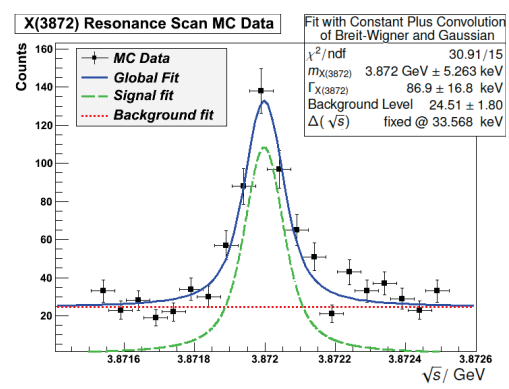

Figure 4: Result of a Monte Carlo simulation performed to check mass determination possibilities of PANDA for $X(3872)$. Each data point corresponds to a change in energy of $500 \mathrm{KeV}$. A four charge pion background has been also included [27].

In the meanwhile, other similar states have also been found in the bottomonium energy range by Belle [30], while the BES III collaboration has discovered another charged charmonium-like axial: the $Z_{C}(3900)$ [31].

Figure 5 shows the proposed layout of the $\overline{\mathrm{P}}$ ANDA experiment. As for GlueX, this is a fixed target detector consisting of a super-conducting Target Spectrometer (TS), surrounding the interaction point, and a Forward Spectrometer (FS), placed within and after a normal conducting dipole. Figure 5 also points out the main components of the apparatus.

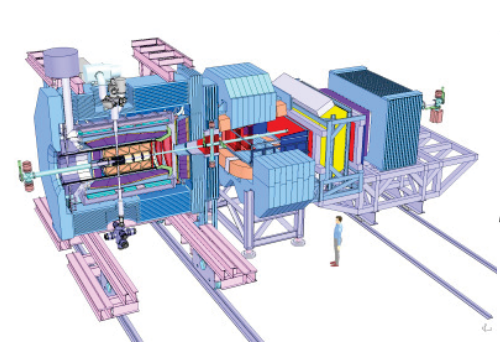

(a)

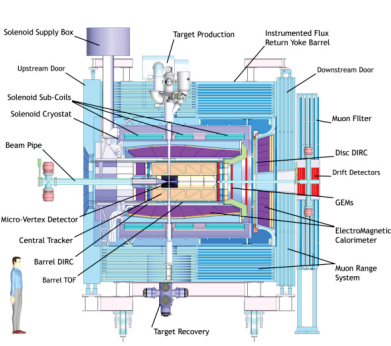

(b)

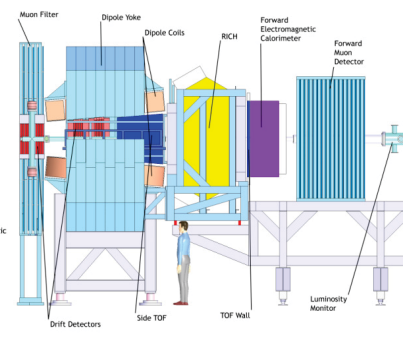

(c)

Figure 5: Layout of the $\bar{P}$ ANDA apparatus (a); detailed view of the Target Spectrometer (TS) (b) and of the Forward Spectrometer (FS) (b).

\section{Conclusions}

Since its foundation, hadron spectroscopy has been trying to confirm the existence of the exotic states predicted by QCD. In the low energy sector, many hints for exotic states have been observed, but the big number of broad overlapping ordinary mesons has not yet allowed to firmly establish none of the candidates.

On the contrary, the recent hadron spectroscopy activity performed at higher energy has allowed to identify some particles whose exotic nature is undisputed. 
The new generation of experiments carried out at the upgraded Jefferson Laboratory and at the new FAIR international center, will help shed new light in this interesting and fundamental field of basic research.

\section{References}

[1] K. G. Wilson Phys. Rev. D 10 2445-2459 (1974).

[2] M. Jacob and G.C. Wick, Ann. Phys. 7, 404 (1959).

[3] B. S. Zou and D. V. Bugg, Eur. Phys. J. A 16, 537 (2003).

[4] D.V. Amelin et al., Phys. At. Nucl. 68, 359 (2005).

[5] G.M. Beladidze et al., Phys. Lett. B 313, 276 (1993).

[6] G.S. Adams et al., Phys. Rev. Lett. 81, 5760 (1998).

[7] E. I. Ivanov et al. Phys. Rev. Lett. 86, 3977 (2001).

[8] S. U. Chung et al., Phys. Rev. D 65, 072001 (2002).

[9] C. A. Baker et al., Phys. Lett. B 563, 140 (2003).

[10] M.G. Alekseev et al., Phys. Rev. Lett. 104, 241803 (2010).

[11] M. Nozar et al., Phys. Rev. Lett. 102, 102002 (2009).

[12] Proposal for JLab Experiment E12-06-102: Mapping the Spectrum of Light Quark Mesons and Gluonic Excitations with Linearly Polarized Photons, http://www.jlab.org/exp_prog/proposals/06/ PR12-06-102.pdf (2006).

[13] D. Lawrence, EPJ Conf. Ser 66, 06011 (2014).

[14] J. J. Dudek et al., Phys. Rev. D 79, 094504 (2009).

[15] J. Beringer et al. (Particle Data Group), Phys. Rev. D 86, 010001 (2012).

[16] S. Olsen, Int. J. Mod. Phys. Conf. Ser. 29 (2014).

[17] S. K. Choi et al., Phys. Rev. Lett. 91, 262001 (2003).

[18] R. Aaij et al. , Phys. Rev. Lett. 110, 222001 (2013).

[19] R. Aaij et al., JHEP 1306, 065 (2013).

[20] S.-K. Choi et al., Phys. Rev. Lett. 91, 262001 (2003).

[21] D. Acosta et al., Phys. Rev. Lett. 93, 072001 (2004).

[22] B. Aubert et al., Phys. Rev. D 71, 071103 (2005).

[23] S.-K. Choi et al., Phys. Rev. D 84, 052004 (2011).

[24] W. Erni et al., arXiv:0903.3905v1.

[25] P. Dalpiaz, Proceedings of Physics With Cooled Low Energetic Antiprotons, edited by H. Poth, 111 (1979).

[26] http://www.fair-center.com/.

[27] M. Galuska et al., PoS BORMIO2012, 018 (2012).

[28] R. Mizuk et al., Phys. Rev. D 80, 031104 (2009).

[29] R. Aaij et al., Phys. Rev. Lett. 112, 222002 (2014).

[30] A. Bondar et al., Phys. Rev. Lett. 108, 122001 (2012).

[31] M. Ablikim et al., Phys. Rev. Lett. 110, 252001 (2013). 